Article

\title{
High Speed Cylindrical Gravitational Collapse with Anisotropic Pressure
}

\author{
Quaid Iqbal ${ }^{1}$, Hasrat Hussain Shah ${ }^{2,3, *}$ (1) and Zahid Ahmad ${ }^{4}$ \\ 1 School of Mathematical Sciences, University of Science and Technology of China, Hefei 230026, \\ Anhui, China; quaid@mail.ustc.edu.cn \\ 2 Key Laboratory for Research in Galaxies and Cosmology, University of Science and Technology of China, \\ Hefei 230026, Anhui, China \\ 3 Department of Mathematical Sciences, Baluchistan University of Information Technology, \\ Engineering and Management Sciences, Quetta 87300, Pakistan \\ 4 Department of Mathematics, COMSATS Institute of Information Technology, University Road, \\ Abbottabad 22060, Pakistan; zahidahmad@ciit.net.pk \\ * Correspondence: hasrat@mail.ustc.edu.cn; Tel.: +86-156-6540-7838
}

Received: 28 April 2018; Accepted: 30 May 2018; Published: 5 June 2018

\begin{abstract}
This paper focuses on the cylindrical symmetric gravitational collapse in the presence of anisotropic fluid. The high speed approximation scheme was used. In this perspective, the effect of anisotropy of pressure in fluid distribution on the collapsing process with the Equation of State (EoS) $p_{t}=\lambda \rho$ and $p_{r}=l \rho,(l+2 \lambda<-1)$. The effect of pressure on collapse in radial and tangential direction was observed for all values of $\lambda$ and $l$. It is determined that, for some values of constants, i.e., $\lambda$ and $l$, collapse results in a Naked Singularity (NS) while, for some values of constants, it does not form NS or Black Hole (BH). This study presents the effect on the collapsing process for all values of $\lambda$ and $l$.
\end{abstract}

Keywords: high-speed; gravitational collapse; anisotropic pressure

\section{Introduction}

The anisotropic and isotropic general relativistic astronomical objects have always been a subject of great interest for researchers in the field astrophysics. The pressure of astronomical objects is supposed to be isotropic, e.g., neutron star is believed to be isotropic in nature. Therefore, Bower and Liang [1] initiated the study of spherical symmetric stellar objects with anisotropic pressure in fluid distribution. The effect of anisotropic pressure on the collapsing process of compact astronomical objects has been studied in [2]. Mak and Harko [3] investigated the solution of spherical symmetric objects with anisotropy of pressure in their fluid distribution. Collapse dynamics of a spherical symmetric star constituted of the dark matter and dark energy with anisotropic pressure have been discussed in [4-6]. The cylindrical symmetric Petrove type D spacetime with naked singularity and matter cloud collapse in the background of anisotropic pressure has been discussed in [7].

Weyl curvature scalar represents the gravitational effects as a tidal disruption field and gravitational radiation. Weyl curvature describing the inhomogeneities of energy density of a spherically symmetric star is examined in [8]. In the collapsing process of an inhomogeneous spherically symmetric star, it is calculated that an inhomogeneity factor may lead the collapse to the formation of Naked Singularity (NS) [9].

Gravitational collapse is considered to be an important issue in general relativity. Gravitational collapse plays a significant role in structural formation in our Universe. The gravitational collapse study as a main source of a gravitational radiation has been a subject of keen interest for astrophysicists. According to the Birkhoffs theorem, in spherically symmetric vacuum spacetimes, there are no 
gravitational wave solutions to the Einstein Field Equations. Another simplest symmetry supposition for a gravitational wave would be cylindrical symmetry. The cylindrical symmetry allows the $1+1$ spacetime of the Einstein Field Equations (EFEs). This type of symmetry may provide a possibility of constructing a model that can demonstrate the nature of interaction between gravitational waves and matter. Oppenheimer and Snyder worked on the phenomenon issue of gravitational collapse in [10]. They considered the Friedmann Robertson Walker (FRW) spacetime geometry. Just after their stupendous contribution in theoretical astrophysics, many models have been studied in order to investigate the gravitational collapse such as spherical symmetric, axisymmetric, cylindrical symmetric with isotropic as well as anisotropic pressure [4,6,11-13]. Nakao and Morisawa [14,15] introduced a an isotropic scheme and investigated cylindrical collapse for dust and perfect fluid, respectively. Cylindrical symmetric gravitational collapse of counter rotating dust shells can be found in the [16,17], axially symmetric vacuum solution [18], and cylindrical symmetric collapse in anisotropic in [7]. An axially symmetric null dust gravitational collapse has been discussed in [19]. The non-spherical gravitational collapse has been discussed in [20-22]. The cylindrical symmetric gravitational collapse has been discussed by considering various models $[23,24]$. The effect of anisotropy in pressure on the gravitational collapse is also a very important phenomenon. We have discussed a number of works about anisotropic pressure above and their effect on the gravitational collapse. Cylindrical symmetric collapse in the background of anisotropic pressure by using matching conditions was investigated in detail anisotropic high speed approximation [25].

We were motivated by the studies given in [14], and generalized the results for anisotropic pressure, whereas the cylindrical symmetric gravitational collapse of type I matter was discussed by applying the principle of anisotropic pressure in fluid distribution [26]. The equation of state has been used to achieve the desired outcomes of pressure effect on gravitational collapse. In our recent study, we have generalized the outcomes of the study given in [14] for anisotropic pressure. We provide the effect of anisotropic pressure on the high speed approximation scheme for all values of the constant used in Equation of State (EoS), while Ahmad and Imtiaz discussed it for only positive values [26]. Finally, we have generalized the results and provided the analytical toy model of high-speed approximation in the background of anisotropic pressure for all values of the constants used in EoS. The spacetime singularity is a consequence of the end state of gravitational collapse. The spacetime singularity is considered to be the most important property of the spacetime [27-29]. However, there are still spacetime singularity speculated, whether it is naked or a $\mathrm{BH}$.

In this study, we extended the results given in [14] for anisotropic pressure. While Ahamd and Imtiaz [26] extend these results for anisotropic pressure, they only focussed on the positive value of $l$ and $\lambda$ that is used in EoS, while we have calculated the results for all values of $l$ and $\lambda$ and proved our results to be consistent with those studies given in [14,26]. This paper has been organized as follows: the field equations for the underlying problem is presented in Section 1. The null dust solution is explained in Section 2. The high-speed approximation scheme is being elaborated in Section 3. The effects of pressure on the high speed collapse are analyzed in Section 4. Finally, a short summary and conclusion are given in Section 5 .

\section{Field Equations}

The metric describing the spacetime geometry inside the collapsing fluid can be cast as: [14]

$$
d s^{2}=e^{2(\gamma-\psi)}\left(-d t^{2}+d r^{2}\right)+e^{2 \psi} d z^{2}+e^{-2 \psi} R^{2} d \phi^{2}
$$

where $R, \psi$ and $\gamma$ are functions of $r$ and $t$ only. The EFEs for metric (1) can be described as 


$$
\begin{aligned}
\dot{\gamma} & =\left(\hat{R}^{2}-\dot{R}^{2}\right)^{-1}\left\{R \hat{R}\left(\dot{\psi}^{2}+\dot{\psi}^{2}\right)-2 R \dot{R} \dot{\psi} \dot{\psi}\right. \\
& \left.+\dot{R} R^{\prime \prime}-\dot{R} \dot{R}^{\prime}-8 \pi G \sqrt{-g}\left(\hat{R} T_{t}^{t}+\dot{R} T_{t}^{r}\right)\right\}, \\
\dot{\gamma} & =-\left(\dot{R}^{2}-\dot{R}^{2}\right)^{-1}\left\{R \dot{R}\left(\dot{\psi}^{2}+\dot{\psi}^{2}\right)-2 R \hat{R} \dot{\psi} \dot{\psi}\right. \\
& \left.+\dot{R} R^{\prime \prime}-\dot{R} \dot{R}^{\prime}-8 \pi G \sqrt{-g}\left(\dot{R} T_{t}^{t}+\dot{R} T_{t}^{r}\right)\right\}, \\
\ddot{\gamma} & -\gamma^{\prime \prime}=\dot{\psi}^{2}-\dot{\psi}^{2}-\frac{8 \pi G}{R} \sqrt{-g} T_{\phi}^{\phi}, \\
\ddot{R} & -R^{\prime \prime}=-8 \pi G \sqrt{-g}\left(T_{t}^{t}+T_{r}^{r}\right), \\
\ddot{\psi} & +\frac{\dot{R}}{R} \dot{\psi}-\psi^{\prime \prime}-\frac{\hat{R}}{R} \dot{\psi}=\frac{-4 \pi G}{R} \sqrt{-g}\left(T_{t}^{t}+T_{r}^{r}\right. \\
& \left.-T_{z}^{z}+T_{\phi}^{\phi}\right),
\end{aligned}
$$

where dot and prime shows the differentiation with respect to $t$ and $r$, respectively. The energymomentum tensor for anisotropic pressure can be expressed as [30]

$$
T_{b}^{a}=\rho u^{a} u_{b}+p_{r} S^{a} S_{b}+p_{t} \Omega_{b}^{a},
$$

where

$$
\Omega_{b}^{a}=\delta_{b}^{a}+u^{a} u_{b}-S^{a} S_{b},
$$

where $p_{t}, p_{r}, \rho, u^{a}$ and $s^{a}$ stands for the tangential and radial pressures, the energy density, 4-velocity and radial vector, respectively. The matter distributed considered in the radial $p_{r}$ and tangential $p_{t}$ direction with positive energy density $\rho$ is consistent with the dominant energy conditions is given by

$$
\rho \geq p_{r}, \quad \rho \geq p_{t} .
$$

The energy-momentum tensor takes the form of dust fluid for $p_{r}=p_{t}=0$, whereas it would be reduced to perfect-fluid for $p_{r}=p_{t}$. Therefore, we are going to generalize the results for anisotropic fluid. In the recent study, we have discussed only two principle stresses in the energy-momentum tensor for the sake of simplicity. In another study, the authors discussed the shear stresses in three directions such as $P_{r}, P_{z}$ and $P_{\phi}[25,31]$. Furthermore this work can extend for three principle stresses used in the energy-momentum tensor. We express the 4-velocity vector and radial vector as

$$
\begin{gathered}
u^{a}=N(1,-1+V, 0,0), \\
s^{a}=N(1-V, 1,0,0),
\end{gathered}
$$

where

$$
N=\frac{e^{(\gamma-\psi)}}{\sqrt{V(2-V)}} .
$$

The variables $D, p_{t}$ and $p_{r}$ introduced in [14] can be expressed as

$$
\begin{aligned}
& D=\frac{\sqrt{-g} N\left(\rho+p_{r}\right)}{\sqrt{V(2-V)}}=\frac{e^{\gamma-\psi} R\left(\rho+p_{r}\right)}{V(2-V)}, \\
& p_{t}=\frac{\sqrt{-g} N p_{t}}{\sqrt{V(2-V)}}=\frac{e^{\gamma-\psi} R p_{t}}{V(2-V)}, \\
& p_{r}=\frac{\sqrt{-g} N p_{r}}{\sqrt{V(2-V)}}=\frac{e^{\gamma-\psi} R p_{r}}{V(2-V)} .
\end{aligned}
$$


For time like $u^{a}, V$ must be positive. From Equations (13)-(15), the energy-momentum tensor (7) can be elaborated as:

$$
T_{b}^{a}=\frac{e^{-3(\gamma-\psi)}}{R}\left[D K^{a} K_{b}+V e^{2(\gamma-\psi)}(2-V)\right.
$$

where

$$
K^{a}=\frac{u^{a}}{N}=(1,-1+V, 0,0)
$$

and

$$
K_{b}=\frac{u_{b}}{N}=e^{2(\gamma-\psi)}(-1,-1+V, 0,0) .
$$

The non-zero components of energy-momentum tensor yields

$$
\begin{aligned}
\sqrt{-g} T_{t}^{t}= & e^{(\gamma-\psi)}\left[-D+V(2-V) p_{t}\right. \\
- & \left.\left(p_{t}-p_{r}\right)\left\{1-(1-V)^{2} e^{-4(\gamma-\psi)}\right\}\right], \\
\sqrt{-g} T_{r}^{t}= & e^{(\gamma-\psi)}(1-V)\left[-D-\left(p_{t}-p_{r}\right)\right. \\
& \left.\left\{1-e^{-4(\gamma-\psi)}\right\}\right]=-\sqrt{-g} T_{t}^{r}, \\
\sqrt{-g} T_{r}^{r}= & e^{(\gamma-\psi)}\left[V(2-V) p_{t}+D(1-V)^{2}\right. \\
- & \left.\left(p_{t}-p_{r}\right)\left\{e^{-4(\gamma-\psi)}-(1-V)^{2}\right\}\right], \\
\sqrt{-g} T_{z}^{z}= & V(2-V) p_{t} e^{(\gamma-\psi)}=\sqrt{-g} T_{\phi^{\prime}}^{\phi}
\end{aligned}
$$

where $g$ is the metric determinant given by Equation (1). The conservation law is expressed by the formula:

$$
T_{b}^{a} ; a=0 .
$$

The above equation can also be written as

$$
T_{b, a}^{a}+\Gamma_{c b}^{a} T_{a}^{c}-\Gamma_{a b}^{d} T_{d}^{a}=0 .
$$

Then, by using Equations (19)-(22), the $t$ and $r$ components of continuity equation can be simplified to:

$$
\begin{aligned}
\partial_{u} D= & \frac{-1}{2}(D V)^{\prime}-\frac{1}{2}\left[( p _ { t } - p _ { r } ) \left\{1-(1-V)^{2}\right.\right. \\
& \left.\left.e^{-4(\gamma-\psi)}\right\}-V(2-V) p_{t}\right]^{\cdot}+\frac{1}{2}\left[\left(p_{t}-p_{r}\right)\right. \\
& \left.(1-V)\left\{1-e^{-4(\gamma-\psi)}\right\}\right]^{\prime}+\frac{V}{2} p_{t}(2-V) \\
& \left(\dot{\psi}-\dot{\gamma}-\frac{\dot{R}}{R}\right)+\frac{D}{2}(1-V)\left[2 \partial_{u}(\psi-\gamma)-\right. \\
& V(\dot{\psi}-\dot{\gamma})]+\frac{\left(p_{t}-p_{r}\right)}{2}\left[e ^ { - 4 ( \gamma - \psi ) } \left\{2 \partial_{u}\right.\right. \\
& \left.(\gamma-\psi)+V\left(\gamma^{\prime}-\psi^{\prime}\right)\right\}+(V-1)\left\{2 \partial_{u}\right. \\
& (\gamma-\psi)-V(\dot{\gamma}-\dot{\psi})\}]
\end{aligned}
$$


and

$$
\begin{aligned}
D \partial_{u} V= & (1-V) \partial_{u} D+\frac{1}{2}\left[(1-V)\left(p_{t}-p_{r}\right)\right. \\
& \left.\left\{1-e^{-4(\gamma-\psi)}\right\}\right]^{\cdot}-\frac{1}{2}\left[p_{t}(2-V) V-\right. \\
& D(1-V) V+\left(p_{t}-p_{r}\right)\left\{(1-V)^{2}-\right. \\
& \left.\left.e^{-4(\gamma-\psi)}\right\}\right]^{\prime}-\frac{D}{2}\left[2 \partial_{u}(\psi-\gamma)-V(\dot{\psi}-\right. \\
& \dot{\gamma})]+\frac{V(2-V) p_{t}}{2}\left(\gamma^{\prime}-\psi^{\prime}+R^{\prime}\right)+ \\
& \frac{\left(p_{t}-p_{r}\right)}{2}\left[\left\{2 \partial_{u}(\gamma-\psi)-V(\dot{\gamma}-\dot{\psi})\right\}-\right. \\
& e^{-4(\gamma-\psi)}(1-V)\left\{2 \partial_{u}(\gamma-\psi)+\right. \\
& \left.\left.V\left(\gamma^{\prime}-\psi^{\prime}\right)\right\}\right],
\end{aligned}
$$

where $v=r+t$ and $u=t-r$ stand for advanced and retarded time, respectively, whereas $2 \partial u=\frac{\partial}{\partial t}-\frac{\partial}{\partial r}$ and $2 \partial v=\frac{\partial}{\partial t}+\frac{\partial}{\partial r}$.

In this study, we discuss the cylindrical symmetric collapse of the fluid in an anisotropic background. We mainly focus on the interior region that was described by Equation (1). We do not focus on the exterior spacetime of the collapsing fluid. Prisco et al. [31] considered the interior spacetime with a general non-rotating matter with anisotropic background, while the exterior is discussed by Einstein-Rosen spacetime. These two spacetimes have been matched by using Darmios matching conditions.

\section{Null Dust Solution}

The very high frequency approximation towards the unidirectional radial unpolarized radiation was explained by null dust solution. This was considered as a valid approximation if the radiation wavelength can be neglected in comparison with the background curvature radius. A number of exact solutions of the EFEs were carried out in the presence of pure null dust [32,33]. When $V \longrightarrow 0$, the solution of the field equations is given below. In this limit, keeping $D, p_{r}$, and $p_{t}$ finite, the energy-momentum tensor (16) is

$$
T_{b}^{a}=\frac{D e^{-3(\gamma-\psi)}}{R} K^{a} K_{b}
$$

where $K^{a}=(1,-1,0,0)$ shows the null vector. This indicates the large collapsing velocity that is $V \approx 0$. The null dust system was considered in our problem for approximation. The deviation $V$ of the 4-velocity from null and use of the linear perturbation method were applied. The collapsing null dust solution can be formulated as:

$$
\begin{aligned}
\psi & =0 \\
R & =r \\
\gamma & =\gamma_{B}(v), \\
8 \pi D G e^{\gamma} & =\frac{d \gamma_{B}}{d v},
\end{aligned}
$$

where $\gamma_{B}(v)$ is a function of $(v)$. For perturbation analysis, the above solution is considered as a background solution. At $r=0$, i.e., the symmetry axis, can be observed from Equations (27) and (29). The energy-momentum tensor diverges if $D$ remains a non-zero value. This implies that at $r=0$ a naked singularity exists. 


\section{High Speed Approximation Scheme}

The linear perturbation analysis for a high speed approximation scheme was applied. For perturbation, we take the null dust solution as a background solution obtained in the previous section.

If $\omega$ is a small parameter, and both $\psi$ and $V$ are of $O(\omega)$, hence, $D, \gamma$ and $R$ are given by

$$
\begin{gathered}
D=\left(1+\delta_{D}\right) D_{B}, \\
e^{\gamma}=\left(1+\delta_{\gamma}\right) e^{\gamma_{B}}, \\
R=\left(1+\delta_{R}\right) r,
\end{gathered}
$$

where $\delta_{D}, \delta_{\gamma}$ and $\delta_{R}$ are also function of $O(\omega)$ and

$$
D_{B}=\frac{1}{8 \pi G e^{\gamma_{B}}} \frac{d \gamma_{B}}{d \nu}
$$

Applying the above conditions stated in Equations (2)-(6) and (26) up to $O(\omega)$, they can be formulated as:

$$
\begin{aligned}
& \delta_{\gamma}^{\prime}=\left(r \delta_{R}\right)^{\prime \prime}+8 \pi G e^{\gamma_{B}} D_{B}\left[\left(\delta_{\gamma}-\psi+\delta_{D}\right)-2 \partial \nu\left(r \delta_{R}\right)\right. \\
& -\frac{2 P_{T} V}{D_{B}}+\left(\frac{p_{t}-p_{r}}{D_{B}}\right)\left\{\left(1+\delta_{\gamma}-\psi\right)-e^{-4 \gamma_{B}}(1\right. \\
& \left.-3 \delta_{\gamma}+3 \psi\right)+2 e^{-4 \gamma_{B}} \partial u\left(r \delta_{R}\right)-2 \partial u\left(r \delta_{R}\right) \\
& \left.\left.+2 V e^{-4 \gamma_{B}}\right\}\right] \text {, } \\
& \ddot{\delta}_{\gamma}=\left(r \delta_{R}\right)^{\prime \prime}+8 \pi G e^{\gamma_{B}} D_{B}\left[\left\{\left(\delta_{\gamma}-\psi+\delta_{D}\right)-2 \partial \nu\left(r \delta_{R}\right)\right.\right. \\
& -V\}+\left(\frac{p_{t}-p_{r}}{D_{B}}\right)\left\{-2 \partial u\left(r \delta_{R}\right)+2 e^{-4 \gamma_{B}} \partial u\left(r \delta_{R}\right)\right. \\
& +\left(1+\delta_{\gamma}-\psi\right)-e^{-4 \gamma_{B}}\left(1-3 \delta_{\gamma}+3 \psi\right) \\
& \left.\left.-V\left(1-e^{-4 \gamma_{B}}\right)\right\}\right] \text {, } \\
& \ddot{\delta}_{\gamma}-\delta_{\gamma}^{\prime \prime}=-\frac{16 \pi G e^{\gamma_{B}}}{r} V p_{t} \\
& \left(r \delta_{R}\right)^{\cdot}-\left(r \delta_{R}\right)^{\prime \prime}=16 \pi G e^{\gamma_{B}} V\left[\left(D_{B}-2 P_{T}\right)\right. \\
& \left.+\left(p_{t}-p_{r}\right)\left(1+e^{-4 \gamma_{B}}\right)\right] \text {, } \\
& \ddot{\psi}-\psi^{\prime \prime}-\frac{\psi^{\prime}}{r}=\frac{8 \pi G e^{\gamma_{B}}}{r} V\left[\left(D_{B}-2 p_{t}\right)\right. \\
& \left.+\left(p_{t}-p_{r}\right)\left(1+e^{-4 \gamma_{B}}\right)\right]
\end{aligned}
$$




$$
\begin{aligned}
\partial u\left(\delta_{\gamma}-\psi+\delta_{D}\right)= & -\frac{1}{2} \frac{d D_{B}}{d v} V-\frac{1}{2}\left(V^{\prime}-V \frac{d \gamma_{B}}{d v}\right) \\
+ & \frac{1}{D_{B}}\left\{\left(p_{t} V\right)^{\cdot}-p_{t} V \frac{d \gamma_{B}}{d v}\right\} \\
- & \frac{1}{2 D_{B}}\left\{V\left(p_{t}-p_{r}\right)\left(1+e^{-4 \gamma_{B}}\right)\right\}^{\prime} \\
- & \frac{1}{D_{B}} \partial u\left[\left(p_{t}-p_{r}\right)-e^{-4 \gamma_{B}}(1\right. \\
- & \left.4 \delta_{\gamma}+4 \psi\right)\left(p_{t}-p_{r}\right)+2 V e^{-4 \gamma_{B}} \\
& \left.\left(p_{t}-p_{r}\right)\right]+\left(\frac{p_{t}-p_{r}}{2 D_{B}}\right)\left[e^{-4 \gamma_{B}}\right. \\
& \left\{2 \partial u\left(\delta_{\gamma}-\psi\right)+V \frac{d \gamma_{B}}{d v}\right\} \\
- & \left.\left\{2 \partial u\left(\delta_{\gamma}-\psi\right)-V \frac{d \gamma_{B}}{d v}\right\}\right] .
\end{aligned}
$$

By applying the above equation, Equation (27) up to $O(\omega)$ yields

$$
\partial u\left\{\left(D_{B}-2 p_{t}\right) V\right\}=\frac{p_{t} V}{r}-\left(1+e^{-4 \gamma_{B}}\right) \partial u\left\{V\left(p_{t}-p_{r}\right)\right\} .
$$

It is interesting to note that our results can be reduced to the perfect fluid for $p_{t}=p_{r}=p$, [14].

\section{Results}

The anisotropic pressure in the energy stresses has been used to observe the effect of anisotropy in pressure on high-speed collapse. For this purpose, we have introduced new variables $\lambda(u, v)$ and $l(u, v)$ as

$$
p_{t}=\rho \lambda(u, v)
$$

and

$$
p_{r}=\rho l(u, v) .
$$

The three energy conditions, which are dominant, strong and weak are considered. The weak energy condition is expressed as:

$$
\rho \geq 0, \rho+p_{r} \geq 0 \text { and } \rho+p_{t} \geq 0 .
$$

The strong energy condition is given as

$$
\rho+p_{r}+2 p_{t} \geq 0
$$

and the dominant energy condition is defined as

$$
\rho \geq\left|p_{r}\right| \text { and } \rho \geq\left|p_{t}\right|
$$

where $\rho$ is positive, all energy conditions are satisfied, if, and only if, for $l$ and $\lambda$, the following relations hold:

$$
-1 \leq l \leq 1,-1 \leq \lambda \leq 1 \text { and } 1+l+2 \lambda \geq 0 .
$$

It is noted that $\lambda$ and $l$ are bounded. From Equations (43) and (44), it follows that

$$
\begin{aligned}
& \frac{p_{r}}{D}=\frac{p_{r}}{p_{r}+\rho}=\frac{l}{l+1}, \\
& \frac{p_{t}}{D}=\frac{p_{t}}{\rho+p_{r}}=\frac{\lambda}{l+1} .
\end{aligned}
$$


From Equations (49) and (42), we get

$$
\begin{aligned}
\partial u\left\{V\left(\frac{1+l-2 \lambda}{l+1}\right)\right\}= & \frac{\lambda V}{(l+1) r}-\left(1+e^{-4 \gamma_{B}}\right) \\
& \partial u\left\{\left(\frac{\lambda-l}{l+1}\right) V\right\} .
\end{aligned}
$$

For $l=-1$, we have observed that the high-speed approximation scheme fails. Hereafter, we assume that $l \neq-1$. For constants $l$ and $\lambda$, Equation (51) becomes

$$
\frac{\partial u V}{V}=\frac{\lambda}{r(1-\lambda)+r e^{-4 \gamma_{B}}(\lambda-l)} .
$$

The final solution of Equation (52) is

$$
V=\text { constant } \times \mathrm{C}_{\mathrm{V}}\left({ }^{\circ}\right) \times \mathrm{r}^{\frac{-2^{\iota}}{\left(1--^{-}\right)+\mathrm{e}^{-4 \mathrm{fl}_{\mathrm{B}\left({ }^{\sim}-1\right)}}}}
$$

where

$$
C_{V}(v)=\exp \left[\{v-U(v)\}^{\left.\frac{-2 \lambda}{(1-\lambda)+e^{-4 \gamma_{B}(\lambda-l)}}\right] .}\right.
$$

According to Equation (53), the velocity perturbation $V$ is infinite for the values $\lambda=l=1$. If the velocity $V$ is infinite, then high speed approximation fails.

For the sake of arbitrariness, when the simultaneous condition of $\lambda \neq l \neq 1$ is considered, the following nuances are taken into account:

5.1. $\lambda=0$ and $\lambda=l$ or $\lambda \neq l$

When $\lambda=0$ and $\lambda=l$ or $\lambda \neq l$, then $1+l+2 \lambda \geq 0$ and, at $r \rightarrow 0$, the velocity perturbation remains finite

$$
V=\text { constant. }
$$

Thus, the high speed approximation may be applied and an NS formed in this case.

5.2. $\lambda \neq 1$ and $\lambda=l$

The case $\lambda \neq 1$ and $\lambda=l$ is divided into the following subcases:

(i) When $0<\lambda<1$ and $\lambda=l$, then $1+l+2 \lambda \geq 0$ and, when $r \rightarrow 0$, the conclusion can be drawn that the velocity $V$ is infinite and the high speed approximation scheme fails.

(ii) When $-1 \leq \lambda<0, \lambda=l$, then initially we check for $\lambda=-1$.

(iii) When $\lambda=-1$ and $\lambda=l$, then $1+l+2 \lambda<0$, whereas $r \rightarrow 0$, then the $V$ is finite and the high speed approximation may work for the above values and an NS is formed.

(iv) When $\frac{-1}{3} \leq \lambda<0$ and $\lambda=l$, then $1+l+2 \lambda \geq 0$ and, when $r \rightarrow 0$, then again the velocity perturbation $V$ is finite and the same result has been obtained.

(v) When $-1<\lambda<\frac{-1}{3}$ and $\lambda=l$, then $1+l+2 \lambda \geq 0$ and, when $r \rightarrow 0$, then the high-speed approximation scheme fails.

(vi) When $\lambda=1$ and $l<\lambda=1 l<1$, then $1+l+2 \lambda \geq 0$, and, when $r \rightarrow 0$, then the $V$ is not finite and the high-speed approximation scheme fails.

5.3. $\lambda \neq 1$ and $\lambda \neq l$

When $\lambda \neq 1$ and $\lambda \neq l$, the following possibilities arise.

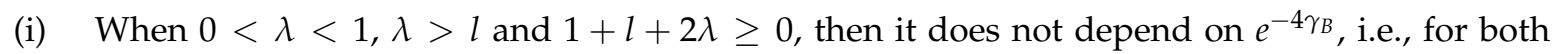
$e^{-4 \gamma_{B}} \geq 1$ and $0<e^{-4 \gamma_{B}}<1$, if $r \rightarrow 0$, then velocity perturbation $V$ becomes infinite and high speed approximation scheme is not applicable. 
(ii) When $0<\lambda<1, \lambda<l$ and it gives us $1+l+2 \lambda \geq 0$, then it depends upon the value of $e^{-4 \gamma_{B}}$. When $e^{-4 \gamma_{B}}>1$ and at $r \rightarrow 0$, it gives us that $V$ is finite, hence the high speed approximation scheme can then be applied; as a result, an NS is formed.

(iii) when $0<e^{-4 \gamma_{B}}<1,0<\lambda<1$ and $\lambda<l$, then $1+l+2 \lambda \geq 0$ and when $r \rightarrow 0$, then the velocity perturbation $V$ becomes infinite and high speed approximation scheme fail.

(iv) When $-1 \leq \lambda<0$ and $\lambda \neq l$. First, we check for $\lambda=-1$ and $l>\lambda$. When $\lambda=-1, l=1$ and $1+l+2 \lambda \geq 0$, the velocity perturbation $V$ depends upon the value of $e^{-4 \gamma_{B}}$, when $0<e^{-4 \gamma_{B}}<1$, and symmetry axis approaches zero i.e., $r \rightarrow 0$, then the velocity perturbation $V$ is finite, hence the same results have been obtained.

In another case, when $\lambda=-1, l=1, e^{-4 \gamma_{B}}>1$ consequently $1+l+2 \lambda \geq 0$ and $r \rightarrow 0$, then the velocity perturbation $V$ becomes infinite and high speed approximation is not applicable. When $\frac{-1}{3} \leq \lambda<0, \lambda<l$, then $1+l+2 \lambda \geq 0$, and, when $r \rightarrow 0$, then the velocity perturbation $V$ is finite, for all values of $0<e^{-4 \gamma_{B}}<12$, and a naked singularity is formed. When $\frac{-1}{3} \leq \lambda<0, \lambda>l$, then the high speed approximation scheme fails.

\section{Conclusions}

Gravitational collapse is a subject of great interest in general theory of relativity. Stupendous research work in this field was carried out by considering different spacetimes and matter fields. Nakao and Morisawa [14,15] initiated the study of a a high speed approximation scheme. They discussed the cylindrical gravitational collapse for dust and perfect fluid, respectively, and interesting outcomes have been carried out. The work done in [14] for perfect fluid is extended by Sharif and Ahmad [34] to two perfect fluids. The anisotropic matter field includes most of the well known physical form of matter like dust and perfect fluid, etc. Hence, it is a more general form of matter than dust and perfect fluid. It would be interesting to discuss gravitational collapse by taking anisotropic pressure in fluid distribution.

Ahmad et al. [35] have discussed spherical collapse using a high speed approximation scheme in the background of anisotropic matter with some useful results. Ahmad and Imtiaz [26] extended this work for cylindrically symmetric spacetime. They have applied the $\operatorname{EoS} l=\sqrt{\frac{p_{r}}{\rho}}$ and $\lambda=\sqrt{\frac{p_{t}}{\rho}}$ for radial and tangential pressure, respectively, to observe the pressure impact on the collapsing process. Their obtained results investigated the effect on the collapse only for positive values of $l$ and $\lambda$.

In this paper, we have extended the results given in [26] for all values of $l$ and $\lambda$ so that they satisfy the energy conditions. We have considered different cases depending upon the values or relations of $l$ and $\lambda$. It is concluded that, for $\lambda=l=1$, the $V$ is infinite and the high speed approximation fails. From the causality requirement, we have considered $\lambda \neq l \neq 1$ at the same time and discussed the following cases i.e., $\lambda=0, \lambda=l$ or $\lambda \neq l, \lambda=l$ and $\lambda \neq 1, \lambda \neq 1, \lambda \neq l$ and $1+l+2 \lambda \geq 0$ in detail. These cases have been divided further into sub cases. For some cases, the high speed approximation scheme fails, whereas, the high-speed approximation scheme work for some cases and the possibility of the formation of NS exists. It is worth mentioning that, for $p_{r}=p_{t}=p$, i.e., $\lambda=l$, our outcomes reduce to the outcomes given in [14].

Author Contributions: Q.I. calculated the results and wrote the first draft.; H.H.S. modified the results and edited the draft and Z.A. review and edit the final version.

Funding: This work is supported by National Natural Science Foundation of China (Grant No. 11725312, $\mathrm{U} 1431228,11233003$, and 11421303).

Acknowledgments: We would like to thank Elena for her careful reading of the paper. We would also like to thank the anonymous reviewers for their valuable comments, which improved the paper significantly.

Conflicts of Interest: The authors declare no conflict of interest. 


\section{Appendix A}

The non-vanishing components of Christoffell symbols of the second kind are

$$
\begin{aligned}
\Gamma_{t t}^{t} & =\gamma^{\prime}-\psi^{\prime}=\Gamma_{r r}^{t}, \\
\Gamma_{t r}^{t} & =\gamma^{\prime}-\psi^{\prime}=\Gamma_{r t}^{t}, \\
\Gamma_{\phi \phi}^{t} & =e^{-2(\gamma-2 \psi)} \psi^{\prime}, \\
\Gamma_{z z}^{t} & =e^{-2 \gamma} R\left(\psi^{\prime} R-R^{\prime}\right), \\
\Gamma_{t t}^{r} & =\gamma^{\prime}-\psi^{\prime}=\Gamma_{r r}^{r}, \\
\Gamma_{t r}^{r} & =\gamma^{\prime}-\psi^{\prime}=\Gamma_{r t}^{r}, \\
\Gamma_{\phi \phi}^{r} & =e^{-2(\gamma-2 \psi)} \psi^{\prime}, \\
\Gamma_{z z}^{r} & =e^{-2 \gamma} R\left(\psi^{\prime} R-R^{\prime}\right)=\Gamma_{t t}^{t}, \\
\Gamma_{\phi t}^{\phi} & =\psi^{\prime}=\Gamma_{t \phi^{\prime}}^{\phi} \\
\Gamma_{\phi r}^{\phi} & =\psi^{\prime}=\Gamma_{r \phi^{\prime}}^{\phi} \\
\Gamma_{t z}^{z} & =-\psi^{\prime}+\frac{R^{\prime}}{R}=\Gamma_{z t \prime}^{z}, \\
\Gamma_{r z}^{z} & =-\psi^{\prime}+\frac{R^{\prime}}{R}=\Gamma_{z r}^{z} .
\end{aligned}
$$

The non-zero components of the Ricci tensor are given as

$$
\begin{aligned}
& R_{t t}=(\dot{\gamma}-\dot{\psi})-(\ddot{\gamma}-\ddot{\psi})-\frac{\ddot{R}}{R}+\frac{\dot{R}}{R}(\dot{\gamma}+\dot{\psi})+\frac{\dot{R}}{R}(\dot{\gamma}-\dot{\psi})-2 \dot{\psi}^{2}, \\
& R_{r r}=-(\dot{\gamma}-\hat{\psi})+(\ddot{\gamma}-\ddot{\psi})-\frac{\dot{R}}{R}+\frac{\dot{R}}{R}(\dot{\gamma}-\dot{\psi})+\frac{\hat{R}}{R}(\dot{\gamma}+\dot{\psi})-2 \dot{\psi}^{2}, \\
& R_{\theta \theta}=(\ddot{\psi}-\dot{\psi}) e^{-2(\gamma-2 \psi)}+\left(\dot{\psi} \frac{\dot{R}}{R}-\dot{\psi} \frac{\hat{R}}{R}\right) e^{-2(\gamma-2 \psi)}, \\
& R_{z z}=e^{-2 \gamma}\left(R \ddot{R}-\dot{\psi} R \dot{R}-\ddot{\psi} R^{2}-R \hat{R}+\dot{\psi} R \hat{R}+\dot{\psi} R^{2}\right), \\
& R_{t r}=-\frac{\dot{R}}{R}+\dot{\gamma} \frac{\dot{R}}{R}+\dot{\gamma} \frac{\dot{R}}{R}-2 \dot{\psi} \dot{\psi} .
\end{aligned}
$$

The Ricci scalar is

$$
\begin{aligned}
R & =\frac{1}{\sqrt{-g}}[-2 \hat{\gamma} R+2 \hat{\psi} R+2 \ddot{\gamma} R-2 \ddot{\psi} R+2 \ddot{R} \\
& \left.+2 \hat{R} \dot{\psi}+2 \dot{\psi}{ }^{2} R-2 \hat{R}-2 \dot{R} \dot{\psi}-2 \dot{\psi}^{2} R\right] .
\end{aligned}
$$

\section{References}

1. Bower, R.L.; Liang, E.P.T. Anisotropic spheres in general relativity. Astrophys. J. 1974,188, 657-665. [CrossRef]

2. Herrera, L.; Santos, N.O. Local anisotropy in self-gravitating systems. Phys. Rep. 1997, 286, 53-130. [CrossRef]

3. Mak, M.K.; Harko, T. Anisotropic stars in general relativity. Proc. R. Soc. Lond. A 2003, 459, 393-408. [CrossRef] 
4. Shah, H.H.; Iqbal, Q. Gravitational collapse of dark matter interacting with dark energy: Black hole formation. Int. J. Mod. Phys. D 2017, 26, 1750142. [CrossRef]

5. Chakraborty, S.; Bandyopadhyay, T. Collapse dynamics of a star of dark matter and dark energy. Gravit. Cosmol. 2010, 16, 151-159. [CrossRef]

6. Shah, H.H. Black hole formation due to collapsing dark matter in a presence of dark energy in the brane-world scenario. Int. J. Mod. Phys. D 2018, 27, 1850020. [CrossRef]

7. Ahmad, F. Cylindrically Symmetric, Asymptotically Flat, Petrov Type D Spacetime with a Naked Curvature Singularity and Matter Collapse. Adv. High Energy Phys. 2017, 2017, 7943649. [CrossRef]

8. Penrose, R.; Hawking, S.W. General Relativity, An Einstein Centenary Survey; Cambridge University Press: Cambridge, UK, 1979.

9. Herrera, L.; Di Prisco, A.; Hernndez-Pastora, J.L.; Santos, N.O. On the role of density inhomogeneity and local anisotropy in the fate of spherical collapse. Phys. Lett. A 1998, 237, 113-118. [CrossRef]

10. Oppenheimer, J.R.; Snyder, H. On Continued Gravitational Contraction. Phys. Rev. 1939, 56, 455-459. [CrossRef]

11. Wang, A. Critical collapse of a cylindrically symmetric scalar field in four-dimensional Einstein's theory of gravity. Phys. Rev. D 2003, 68, 064006. [CrossRef]

12. Cai, R.-G.; Wang, A. Black hole formation from collapsing dust fluid in a background of dark energy. Phys. Rev. D 2006, 73, 063005. [CrossRef]

13. Ahmad, Z.; Shah, H.H. Gravitational Collapse of Dust Cloud with Dark Energy. Int. J. Theor. Phys. 2013, 52, 1490-1503. [CrossRef]

14. Nakao, K.; Morisawa, Y. High-Speed Cylindrical Collapse of Perfect Fluid. Prog. Theor. Phys. 2005, 113, 73-85. [CrossRef]

15. Nakao, K.; Morisawa, Y. High speed dynamics of collapsing cylindrical dust fluid. Class. Quantum Gravity 2004, 21, 2101. [CrossRef]

16. Goncalves, S.; Jhingan, S. A NOTE ON THE CYLINDRICAL COLLAPSE OF COUNTER-ROTATING DUST. Int. J. Mod. Phys. D 2002, 11, 1469. [CrossRef]

17. Periera, P.R.C.T.; Wang, A. Gravitational collapse of cylindrical shells made of counterrotating dust particles. Phys. Rev. D 2000, 62, 124001. [CrossRef]

18. Sarma, D.; Ahmed, F.; Patgiri, M. Axially Symmetric, Asymptotically Flat Vacuum Metric with a Naked Singularity and Closed Timelike Curves. Adv. High Energy Phys. 2016, 2016, 2546186. [CrossRef]

19. Ahmed, F. Axially Symmetric Null Dust Space-Time, Naked Singularity, and Cosmic Time Machine. Adv. High Energy Phys. 2017, 2017, 3587018. [CrossRef]

20. Chiba, T. Cylindrical Dust Collapse in General Relativity: Toward Higher Dimensional Collapse. Prog. Theor. Phys. 1996, 95, 321-338. [CrossRef]

21. Senovilla, J.M.M.; Vera, R. Cylindrically symmetric dust spacetime. Class. Quantum Gravity 2000, 17, 2843. [CrossRef]

22. Bondi, H. The mass of cylindrical systems in general relativity. Proc. R. Soc. Lond. A 1990, 427, $259-264$. [CrossRef]

23. Chakraborty, S.; Chakraborty, S. Gravitational collapse of dissipative fluid as a source of gravitational waves. Ann. Phys. 2016, 364, 110-119. [CrossRef]

24. Mahmood, T.; Shah, S.M.; Abbas, G. Gravitational collapse and expansion of charged anisotropic cylindrical source. Astrophys. Space Sci. 2015, 357, 1. [CrossRef]

25. Herrera, L.; le Denmat, G.; Marcilhacy, G.; Santos, N.O. Static cylindrical symmetry and conformal flatness. Int. J. Mod. Phys. D 2005, 14, 657-666. [CrossRef]

26. Ahmad, Z.; Imtiaz, B. High-Speed Cylindrical Collapse of Type-I Matter. Braz. J. Phys. 2013, 43, 57-63. [CrossRef]

27. Penrose, R. Gravitational Collapse and Space-Time Singularities. Phys. Rev. Lett. 1965, 14, 57-59. [CrossRef]

28. Hawking, S.W. The occurrence of singularities in cosmology. III. Causality and singularities. Proc. R. Soc. Lond. 1967, 300, 187-201. [CrossRef]

29. Hawking, S.W.; Penrose, R. The singularities of gravitational collapse and cosmology. Proc. R. Soc. Lond. 1970, 314, 529-548. [CrossRef]

30. Hawking, S.W.; Ellis, G.F.R. The Large Scale Structre of Spacetime; Cambridge University Press: Cambridge, UK, 1973. 
31. Di Prisco, A.; Herrera, L.; MacCallum, M.A.H.; Santos, N.O. Shearfree cylindrical gravitational collapse. Phys. Rev. D 2009, 80, 064031. [CrossRef]

32. Kramer, D.; Stephani, H.; MacCallum, M.A.H.; Herlt, E. Exact Solutions of Einsteins Field Equation; Cambridge University Press: Cabridge, UK, 1980.

33. Bičák, J.; Kuchař, K. Null dust in canonical gravity. Phys. Rev. D 1997, 56, 4878-4895.

34. Sharif, M.; Ahmad, Z. High-speed cylindrical collapse of two perfect fluids. Gen. Relativ. Gravit. 2007, 39, 1331-1344. [CrossRef]

35. Ahmad, Z.; Harada, T.; Nakao, K.; Sharif, M. High-speed collapse of a hollow sphere of type I matter. Class. Quantum Gravity 2009, 26, 035007. [CrossRef]

(C) 2018 by the authors. Licensee MDPI, Basel, Switzerland. This article is an open access article distributed under the terms and conditions of the Creative Commons Attribution (CC BY) license (http://creativecommons.org/licenses/by/4.0/). 